\title{
Palmeras utilizadas por los indígenas Yuracaré del Territorio Indígena Parque Nacional Isiboro- Sécure (Cochabamba, Bolivia)
}

Palms used by the Yuracaré of the Indigenous Territory National Park IsiboroSécure (Cochabamba, Bolivia)

Palmiers utilisés par les Yuracaré du Territoire Indigène du Parc National Isiboro-Sécure (Cochabamba, Bolivie)

Fabiola Montoya M. y Mónica Moraes R.

\section{OpenEdition}

\section{Journals}

Edición electrónica

URL: http://journals.openedition.org/ethnoecologie/1697

DOI: $10.4000 /$ ethnoecologie. 1697

ISSN: $2267-2419$

Editor

Laboratoire Eco-anthropologie et Ethnobiologie

Referencia electrónica

Fabiola Montoya M. et Mónica Moraes R., «Palmeras utilizadas por los indígenas Yuracaré del Territorio Indígena Parque Nacional Isiboro-Sécure (Cochabamba, Bolivia) », Revue d'ethnoécologie [En ligne], 5 | 2014, mis en ligne le 30 juin 2014, consulté le 19 avril 2019. URL : http:// journals.openedition.org/ethnoecologie/1697; DOI : 10.4000/ethnoecologie.1697

Este documento fue generado automáticamente el 19 abril 2019.

Licence Creative Commons

Revue d'ethnoécologie est mis à disposition selon les termes de la licence Creative Commons

Attribution - Pas d'Utilisation Commerciale - Pas de Modification 4.0 International. 


\title{
Palmeras utilizadas por los indígenas Yuracaré del Territorio Indígena Parque Nacional Isiboro-Sécure (Cochabamba, Bolivia)
}

\author{
Palms used by the Yuracaré of the Indigenous Territory National Park Isiboro- \\ Sécure (Cochabamba, Bolivia) \\ Palmiers utilisés par les Yuracaré du Territoire Indigène du Parc National \\ Isiboro-Sécure (Cochabamba, Bolivie)
}

Fabiola Montoya M. y Mónica Moraes R.

\section{Introducción}

1 Las palmeras ofrecen diversos usos para las comunidades humanas, esto hace que la vida especialmente en aéreas rurales esté estrechamente ligada a estas plantas (Moraes et al. 2014; Borchsenius \& Moraes 2006; Paniagua 2005a; Moraes 2004a, b; Vormisto 2002). Esta familia es un grupo que presta diversos servicios al hombre; gran parte de la vida cotidiana de los grupos indígenas depende de las palmeras (Galeano \& Bernal 2010; Moraes \& Velarde 2009; Paniagua et al. 2007; Paniagua 2005b) y en el caso de Bolivia se han registrado a 58 especies (que representan el $64 \%$ del total) con alguna categoría de uso (Moraes 2014). En el área de influencia del sitio evaluado en el presente estudio se ha documentado un total de quince palmeras útiles: Astrocaryum gratum, Attalea blepharopus, A. princeps, Bactris major, B. chaveziae, B. gasipaes, Chamaedorea angustisecta, Euterpe precatoria , Geonoma brongniartii, G. deversa, Hyospathe elegans, Iriartea deltoidea, Mauritia flexuosa, Oenocarpus bataua y Socratea exorrhiza (Paniagua et al. 2010).

Los bosques amazónicos han sido habitados por grupos humanos que han desarrollado un modo de vida según su entorno y aprendieron durante años a utilizarlos de manera 
sostenible (Medinaceli \& Aguirre 2006; Bocco et al. 2000; Martin 2000; Vargas 1997; Cerón 1995). El ser humano es un elemento del ecosistema y por ello es clave tomar en cuenta la importancia de sus conocimientos y uso tradicional que afectan el uso y manejo de especies y ecosistemas (Pardo de Santayana \& Gómez Pellón 2003); pues desarrollan su civilización basados en el conocimiento de la naturaleza, los pisos ecológicos y la variación del clima, favoreciendo al desarrollo de la tecnología para la diversificación de los principales recursos alimenticios y medicinales (Rivera 2006). Actualmente estos pueblos sostienen que la diversidad es la clave para la producción de alimentos y plantas medicinales (Rivera 2006; Bocco et al. 2000; Posey 2000); han dependido de los componentes de la vegetación para solucionar la mayoría de sus necesidades vitales (Pardo de Santayana \& Gómez Pellón 2003).

3 Sin embargo, el proceso de colonización acelerado y no planificado va produciendo una progresiva transculturización de las sociedades (Aguirre 2006; Quintana \& Vargas 1995). Además existen diferentes factores socioeconómicos (género, etnicidad, edad, educación, accesibilidad, migración, entre otros) y ecológicos que afectan el conocimiento y uso tradicional de las plantas; estos factores pueden actuar de forma dependiente o interactuando provocando la pérdida o conservación de los conocimientos (Apaza 2008; Paniagua et al. 2007; Amaya 2005; Campos \& Ehringhaus 2003).

Desde la segunda mitad del siglo XIX, empezó la colonización del Chapare (zona tropical del departamento de Cochabamba, Bolivia) formándose varios pueblos; entre ellos el pueblo étnico de los Yuracaré fue desplazado a tierras más difíciles de habitar (Cardoso 2002 ; CONIYURA 1998). Según estas fuentes, fueron empleados como peones en la apertura de caminos, construcción de puentes y cazadores-pescadores por los colonizadores Quechua. Los Yuracaré - que pertenecen a la familia lingüística YuracaréMoxeña en Bolivia- constituyen un pueblo indígena ribereño con una población de alrededor a 3.440 personas; según el censo realizado el 2004 por Conniob (Confederación Nacional de Nacionalidades Indígenas y Originarias de Bolivia). Se los considera un pueblo cristianizado y en las últimas décadas han perdido mucho de sus aspectos culturales, cosmogónico, mitológico y de creencias ancestrales que formaban parte de su identidad (Cardoso 2002). Los colonos, cazadores furtivos, cocaleros y madereros amenazan su territorio, debido a la explotación de los recursos naturales provocando su disminución (Cardoso 2002).

5 Las evaluaciones etnobotánicas procuran el rescate de información acerca del uso y manejo de recursos vegetales por los grupos humanos que han vivido con la naturaleza por varias generaciones; registran el valor de la información obtenida de los indígenas poseedores de conocimiento sobre la naturaleza que les rodea, así la información ayudara a mejorar y pulir los trabajos en la operación de algún plan de manejo (Martin 2000). La presente investigación etnobotánica fue realizada en el marco del proyecto "Impactos de cosecha de palmeras en bosques tropicales (PALMS)" (2009-2013) financiado por el Séptimo Programa de la Unión Europea en su grupo de trabajo (WP3): Productos de palmeras útiles para la población local. El objetivo principal de este estudio fue documentar las especies de palmeras útiles para las comunidades Yuracaré, evaluar comparativamente las especies y las categorías de uso y comparar el conocimiento y uso de las palmeras según el género, edad y la comunidad a la que pertenece cada entrevistado. 


\section{Área de estudio}

El Parque Nacional Isiboro Sécure fue creado en 1990 y se reconoció como territorio de los pueblos Mojeño, Yuracaré y Tsimane' por lo que se lo denominó Territorio Indígena Parque Nacional Isiboro Sécure (o TIPNIS) (Sernap 2002, Subcentral Tipnis 2010). Está ubicado entre los departamentos de Beni (Provincia Moxos) y de Cochabamba (Provincia Chapare) al centro de Bolivia. Se encuentra entre $65^{\circ} 04^{\prime}-66^{\circ} 40^{\prime}$ Longitud Oeste y $15^{\circ} 27^{\prime}$ $16^{\circ} 47^{\prime}$ Latitud Sur (Sernap 2002) y su rango altitudinal oscila entre $180-3.000 \mathrm{~m}$ de altitud, con un promedio de 300-4.000 m (Killeen et al. 1993; Sernap 2002)

Actualmente el TIPNIS (Figura 1) cuenta con una extensión de 1.091.656 ha, se encuentra entre la llanura beniana y las sierras subandinas de Cochabamba; en la subcuenca amazónica del Río Mamoré, representada por uno de sus afluentes principales que es el Río Securé (Sernap 2002; Killeen et al. 1993). La ubicación del Tipnis es típicamente transicional a partir de un gradiente de cuatro pisos ecológicos, desde las zonas de Yungas lluviosos hasta la llanura aluvial baja del norte, sujeta a fuertes inundaciones estacionales (Sernap 2002, Subcentral Tipnis 2010).

Figura 1 : Área de estudio y comunidades Yuracaré estudiadas, señaladas con círculos rojos.

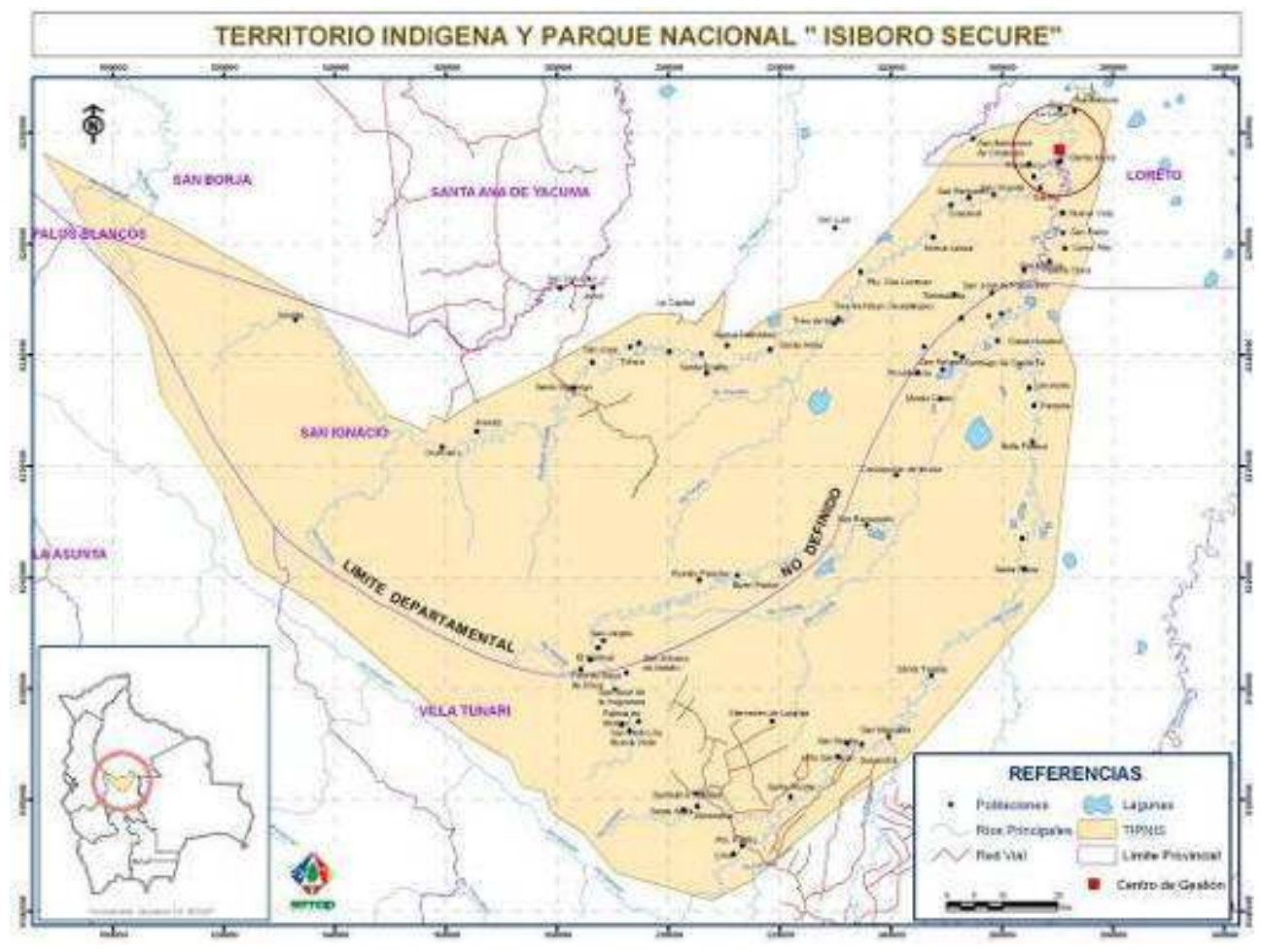

Source : www.sernap.gov.bo

Las temperaturas medias anuales van desde los $15^{\circ} \mathrm{C}$ hasta los $32^{\circ} \mathrm{C}$ (Sernap 2002; Hinojosa et al. 2001; Killeen et al. 1993). Los valores de precipitación anual fluctúan según las zonas, variando desde $1.900 \mathrm{~mm}$ hasta los $3.500 \mathrm{~mm}$, media anual (Sernap 2002). 


\section{Métodos}

9 Esta investigación etnobotánica se basa en el protocolo propuesto por Paniagua et al. (2010). Para la selección de los informantes se utilizaron los datos del censo de población realizado por Paniagua et al. (2010), tomando solamente a informantes mayores a los 18 años. Esta investigación se guía según las bases establecidas por Martin (2000), en relación a la observación participante. Esta metodología se aplica mediante la participación de las actividades cotidianas de la gente de la comunidad, aprovechamiento de las situaciones espontáneas, visitas inesperadas a sus casas, en actividades de la cocina antes de cada comida, o acompañando a los chacos, entre otras actividades para conseguir la confianza de ellos y así poder obtener datos fiables (Martin 2000). Además se realizaron entrevistas semi-estructuradas y colecciones botánicas de las palmeras.

\section{Visita a las comunidades}

10 De un total aproximado de veinte comunidades Yuracaré, se seleccionaron cinco comunidades de trabajo que están en el área de influencia del Tipnis, cada comunidad elegida tiene más de veinte personas adultas que es el número mínimo de entrevistas por comunidad para completar la muestra. El trabajo de campo se realizó en dos campañas durante 2-4 semanas en 2011. En cada comunidad se llevó a cabo un taller comunal en el cual se reunió a más del $60 \%$ de la población adulta de las comunidades para explicar el tipo de trabajo, sus objetivos y alcances; además se seleccionó a los informantes clave, que son personas que poseen mayor experiencia y conocimiento (Martin 2000). Estos informantes fueron seleccionados por la comunidad, ya que entre ellos saben quiénes son los que tienen mayor conocimiento del monte y las palmeras.

\section{Entrevistas y colectas botánicas}

11 Se trabajó con dos tipos de informantes: Los expertos quienes son hombres y mujeres con mayor conocimiento sobre el uso de las palmeras. El tiempo empleado para el trabajo fue de un día completo, el cual incluye la búsqueda de palmeras, la entrevista y la colección de especímenes. Por otro lado, se trabajó con informantes en general, quienes proporcionaron información acerca de la variación en el conocimiento de uso de las palmeras en la comunidad; estas entrevistas fueron de una hora.

12 Para evaluar las diferencias en el conocimiento sobre el uso de las palmeras por género, se consideró una proporción del 50\% informantes hombres y 50\% mujeres. Para evaluar la relación entre la edad y el conocimiento de uso de las palmeras, los individuos entrevistados fueron divididos en tres categorías de edad: 18-40 años, de 41-60 y más de 60 años; buscando reflejar la propia organización social de la comunidad Yuracaré.

Las entrevistas que se realizaron para conocer los diferentes usos de las palmeras fueron de tipo semiestructuradas, ya que permiten tener una mayor amplitud de respuesta (Martin 2000). Para el registro de usos, éstos fueron organizados en diez categorías y subcategorías etnobotánicas. Para cada uso se registró la parte de la palmera empleada. Los datos se registraron por escrito, en las entrevistas se mantuvo expresiones y palabras nativas con el fin de generar mayor confianza (Martin 2000). 
14 Al realizar la colecta botánica, se registraron datos de la palma como el nombre local, altura, DAP, hábito, medida de la vaina, peciolo, raquis y el total de la hoja, el ancho basal, medial y apical y el número de pinnas o foliolos. Se tomaron las medidas de tres hojas diferentes de cada individuo y fueron prensadas en campo, para su determinación taxonómica y depósito en el Herbario Nacional de Bolivia (LPB) en La Paz.

\section{Interpretación de datos}

Para comparar la edad entre comunidades y por género, se recurrió a una ANOVA bifactorial y para comparar las proporciones de géneros entre comunidades se uso una prueba de Chi-cuadrado. En cuanto al análisis del número de palmeras útiles por género, se aplicó la prueba de U de Mann-Whitney, para comparar comunidad y categoría de edad se uso el test de Kruskal Wallis. En los casos donde se encontró diferencia significativa se utilizó la prueba U de Mann-Whitney con el fin de contrastar grupos.

Respecto a la riqueza y diversidad de uso de palmeras, se trabajó con los siguientes parámetros: Número de especies reconocidas como aprovechables por cada grupo, diversidad cuantificada mediante el índice inverso de Simpson (Cinv) - donde se usa la proporción de aparición en las entrevistas - como la abundancia relativa de la especie y finalmente composición de la comunidad; los cuales se muestran en los gráficos rango abundancia. Además se realizó una prueba estadística para comparar índices de diversidad:

$\mathrm{t}=\mathrm{H}_{1}^{\prime}-\mathrm{H}_{2}^{\prime} / \mathrm{s} \mathrm{H}_{1}^{\prime}-\mathrm{H}_{2}^{\prime}$, donde, $\mathrm{H}^{\prime}$ = índice de diversidad de Shannon-Wiener y la hipótesis nula es que la diversidad es la misma en ambos grupos, dado que la prueba solo permite comparar dos grupos se realizaron varias combinaciones.

17 También se halló el valor de uso de cada palmera para los entrevistados de las diferentes comunidades mediante la metodología propuesta por Phillips \& Gentry (1993), donde se tiene la siguiente fórmula:

$\mathrm{UV}_{\mathrm{n}}=\Sigma \mathrm{U}_{\mathrm{ni}} / \mathrm{n}_{\mathrm{t}}$ " donde, $\mathrm{UV}_{\mathrm{n}}=\mathrm{el}$ "valor de uso de la especie n" que resulta de la sumatoria del uso de la especie para cada informante $\left(\Sigma U_{\text {ni }}\right)$ entre el número total de informantes.

\section{Resultados}

Se entrevistó un total de 106 personas pertenecientes a cinco comunidades Yuracaré: San Antonio, Sesejcsama, San Juan del Isiboro, San Nandita y San Benito, distribuidas en género y edad (Tabla 1).

Tabla 1 : Población total y participantes; distribución de género y edad en comunidades Yuracaré.

\begin{tabular}{|c|c|c|c|c|c|c|c|}
\hline Comunidad & Coordenadas geográficas & Población total & Población participante & $\mathbf{F}$ & Edad & M & Edad \\
\hline Sesecjsama & $16^{\circ} 37^{\prime} 52^{\prime \prime S}, 65^{\circ} 44^{\prime} 23^{\prime \prime O}$ & 40 & 24 & 15 & 33.93 & 9 & 37 \\
\hline San Antonio & $16^{\circ} 21^{\prime} 17^{\prime \prime} \mathrm{S}, 65^{\circ} 56^{\prime} 23^{\prime \prime} \mathrm{O}$ & 43 & 23 & 12 & 29.42 & 11 & 34.73 \\
\hline San Benito & $16^{\circ} 32^{\prime} 10^{\prime \prime} \mathrm{S}, 65^{\circ} 30^{\prime} 16^{\prime \prime} \mathrm{O}$ & 39 & 24 & 12 & 38.17 & 12 & 34.75 \\
\hline San Juan & $16^{\circ} 32^{\prime} 40^{\prime \prime} \mathrm{S}, 65^{\circ} 30^{\prime} 16^{\prime \prime O}$ & 24 & 14 & 6 & 32.83 & 8 & 31.88 \\
\hline San Nandita & $16^{\circ} 32^{\prime} 37^{\prime \prime S}, 65^{\circ} 30^{\prime} 08^{\prime \prime O}$ & 27 & 21 & 6 & 27.17 & 15 & 35.4 \\
\hline
\end{tabular}

$\mathrm{F}=$ femenino, $\mathrm{M}=$ masculino 
19 En promedio cada entrevistado identificó entre 5-6 palmeras útiles. Las comunidades de
San Juan, San Benito y San Antonio reportaron seis, en cambio para Sesejcsama y San
Nandita fueron cinco. En cuanto al conocimiento de palmeras útiles por género, no existe diferencia significativa entre hombres y mujeres.

De acuerdo a la prueba estadística U de Mann-Whitney, la comunidad de Sesejcsama es la que presenta mayor diferencia respecto a las otras en cuanto al número de palmeras útiles (Tabla 2); existe diferencia significativa con todas las comunidades, excepto con San Nandita. Según el índice de Simpson, la comunidad con mayor diversidad en palmeras es San Antonio (Cinv= 8.85, tabla 3) con 12 palmeras, seguido por San Nandita ( $C i n v=7.32$, 57\%) y la comunidad con menor diversidad es Sesecjsama (Cinv= 6.02). En general, las curvas de rango-abundancia (Figuras $2 \mathrm{a}$ a $2 \mathrm{e}$ ) no muestran a una especie dominante. Sin embargo, las curvas que representan a San Antonio y San Benito tienen a Bactris gasipaes (tëmbi ${ }^{1}$, en lengua Yuracaré) como la más abundante (Figuras 2a y 2b). El resto de las curvas registran a otras especies como más abundantes, sin embargo todas están seguidas por B. gasipaes. La curva que representa a Sesejcsama tiene a Iriartea deltoidea (winna ${ }^{2}$ ) como la más abundante (Figura 2c), para San Nandita es Attalea blepharopus (corokke³, Figura 2d) y para San Juan, Astrocaryum gratum (choo ${ }^{4}$, Figura 2e).

Tabla 2 : Prueba de U de Mann-Whitney para el número de palmeras útiles entre comunidades Yuracaré $\left({ }^{*} p<0.05\right)$.

\begin{tabular}{|l|c|c|c|c}
\hline Comunidades & Sesecjsama & San Benito & San Nandita & San Juan \\
\hline San Antonio & $\mathrm{U}=181 ; \mathrm{p}=0.027^{\star}$ & $\mathrm{U}=275 ; \mathrm{p}=0.982$ & $\mathrm{U}=195.5 ; \mathrm{p}=0.263$ & $\mathrm{U}=5142.5 ; \mathrm{p}=0.567$ \\
\hline Sesecjsama & & $\mathrm{U}=163.5 ; \mathrm{p}=0.008^{\star}$ & $\mathrm{U}=193 ; \mathrm{p}=0.158$ & $\mathrm{U}=79.5 ; \mathrm{p}=0.006^{\star}$ \\
\hline San Benito & & & $\mathrm{U}=190 ; \mathrm{p}=0.140$ & $\mathrm{U}=141 ; \mathrm{p}=0.427$ \\
\hline San Nandita & & & & $\mathrm{U}=90.5 ; \mathrm{p}=0.056$ \\
\hline
\end{tabular}

Tabla 3 : Riqueza e índice inverso de Simpson (Cinv) por comunidad.

\begin{tabular}{l|c|c|}
\hline Comunidad & Riqueza & C(inv) \\
\hline San Antonio & 12 & 8.85 \\
\hline Sesecjsama & 8 & 6.02 \\
\hline San Benito & 10 & 7.17 \\
\hline San Nandita & 8 & 7.32 \\
\hline San Juan & 9 & 7.04 \\
\hline
\end{tabular}


Figura 2 : Curvas rango-abundancia: a. San Antonio. b. San Benito. c. Sesecjsama. d. San Nandita. e. San Juan.
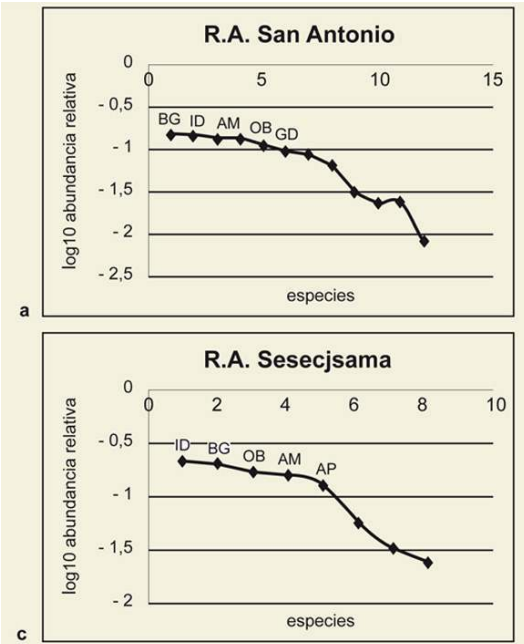
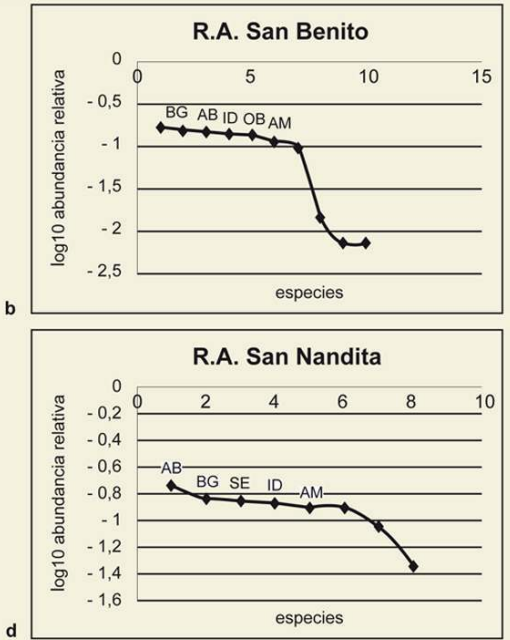

d

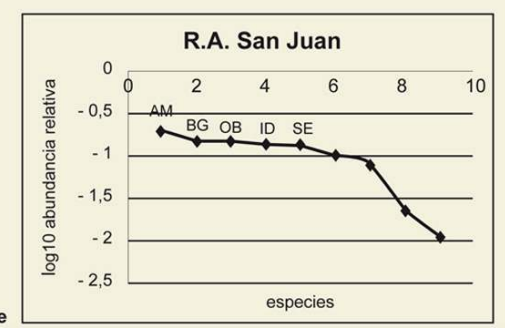

$\mathrm{BG}=$ Bactris gasipaes, $\mathrm{ID}=$ Iriartea deltoidea, $\mathrm{AM}=$ Astrocaryum gratum, $\mathrm{OB}=$ Oenocarpus bataua, $\mathrm{GD}=$ Geonoma deversa, $\mathrm{AB}=$ Attalea blepharopus, $\mathrm{AP}=$ Attalea princeps, $\mathrm{SE}=$ Socratea exorrhiza

Las personas mayores a los 60 años pudieron identificar más de siete palmeras útiles; mientras que las menores a los 60 años reportaron seis. Según la prueba U de MannWhitney, existe diferencia significativa entre las personas mayores a los 60 años que reconocieron un número mayor de palmeras útiles a diferencia de las otras dos categorías de edad (Tabla 4).

Los Yuracaré utilizan catorce especies de palmeras (Tabla 5) y se encuentran en 1-5 categorías. El número de registros en porcentaje, o las veces que fue mencionada cierta palmera para diferentes usos es mayor para I.deltoidea y B. gasipaes con $16.6 \%$ cada una (Figura 3), seguida por A. gratum (14.6\%) y Oenocarpus bataua (uruppa ${ }^{5}, 13.7 \%$ ). El 49\% mencionó como palmera más valiosa a A. blepharopus, a ésta le sigue I. deltoidea (28\%) (Figura 4).

Tabla 4 : Prueba de U de Mann-Whitney para el número de palmeras útiles entre categoría de edad $\left({ }^{\star} \mathrm{p}<0.05\right)$.

\begin{tabular}{c|c|c} 
Edad & De 40 a 60 & Más de 60 \\
\hline De 18 a 40 & $U=913 ; p=0.337$ & $U=27.5 ; p=0.005^{*}$ \\
\hline De 40 a 60 & & $U=13 ; p=0.011^{*}$ \\
\hline
\end{tabular}


Tabla 5 : Palmeras usadas por las cinco comunidades Yuracaré. Se registra el porcentaje de presencia por especie en 106 entrevistas.

\begin{tabular}{|c|c|c|c|c|c}
\hline Nombre cientifico & Nombre Yuracaré & Nombre castellano & Registros (\%) & Usos & Categorias de uso \\
\hline Aiphanes horrida & kunna & Chontilla & $4(0.7)$ & 1 & 1 \\
\hline Astrocaryum gratum & choo & Chonta & $88(14.6)$ & 3 & 4 \\
\hline Attalea blepharopus & corokke & Palla & $54(8.9)$ & 6 & 5 \\
\hline Attalea princeps & sipe & Motacú & $66(10.9)$ & 5 & 6 \\
\hline Bactris gasipaes & tëmbi & Tembé & $100(16.6)$ & 7 & 4 \\
\hline Bactris major & tërishë14 & Marayahú grande & $4(0.7)$ & 2 & 2 \\
\hline Chamaedorea angustisecta & siyeye ${ }^{15}$ & Siyaya & $1(0.2)$ & 1 & 1 \\
\hline Euterpe precatoria & murishi & Asaí & $4(0.7)$ & 3 & 3 \\
\hline Geonoma deversa & mushshu & Jatata & $22(3.6)$ & 1 & 1 \\
\hline Hyospathe elegans & Siribare & & $8(1.4)$ & 1 & 1 \\
\hline Iriartea deltoidea & Winna & Pachuba & $100(16.6)$ & 8 & 5 \\
\hline Mauritia flexuosa & shushundala & Palma real & $9(1.5)$ & 1 & 1 \\
\hline Oenocarpus bataua & Uruppa & Majo & $83(13.7)$ & 4 & 3 \\
\hline Socratea exorrhiza & putërëjtë & Pachubilla & $61(10.1)$ & 6 & 5 \\
\hline
\end{tabular}

Figura 3 : Porcentaje de registros para especies nativas de palmeras según diferentes usos.

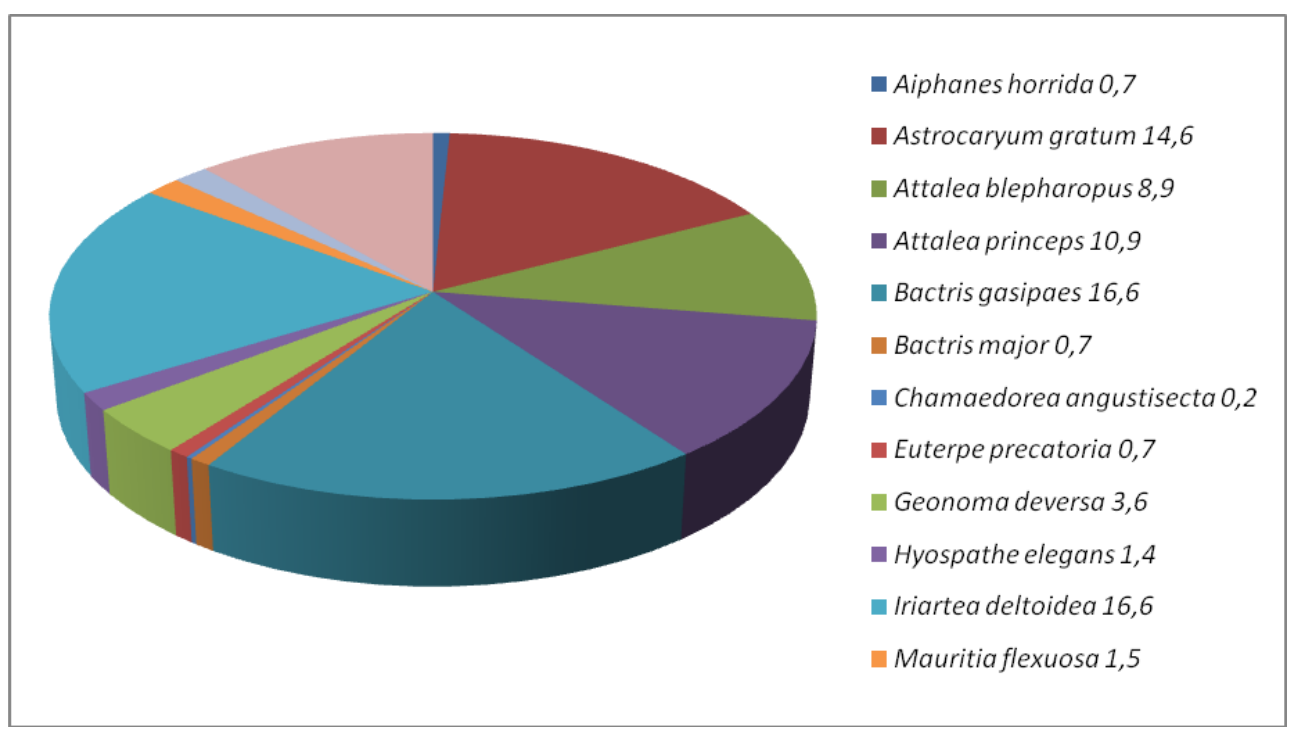

Figura 4 : Especie más valiosa según los entrevistados.

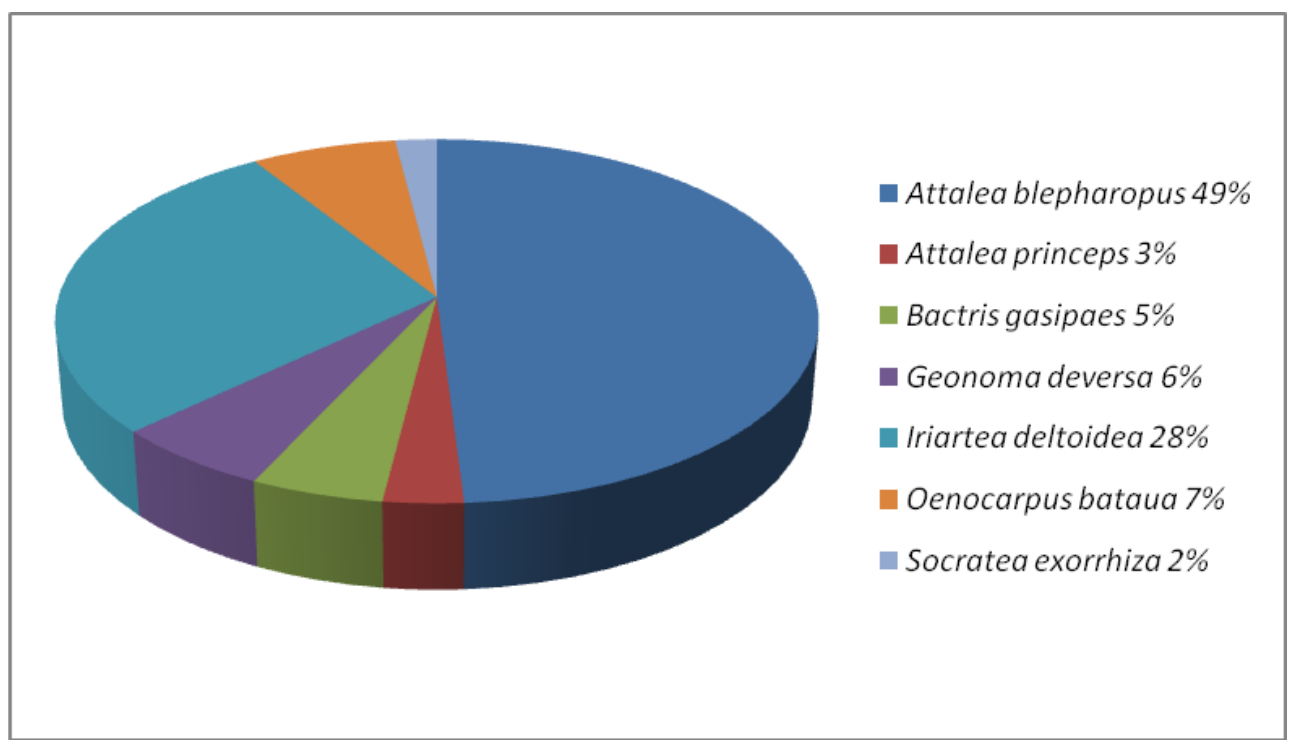


Las especies con el mayor valor de uso son A. blepharopus (2.2), I. deltoidea (2.1) y 0. bataua (2) (Tabla 6) y se encuentran en las categorías construcción, alimenticia, utensilios y herramientas, siendo las tres más importantes. Calculando los valores de uso por comunidad Yuracaré, B. gasipaes registra mayor valor de uso (2.1) en San Antonio y Sesecjsama (V.U.= 2.8). Attalea blepharopus y A. princeps $\left(\right.$ sipe $\left.^{6}\right)$ presentan valores de uso más altos en las demás comunidades: A. blepharopus en San Benito y San Nandita con 3.1 y A. princeps en San Juan con 3.3. Iriartea deltoidea está entre los mayores valores de uso en casi todas las comunidades (todos mayores a 2) excepto en San Antonio; lo mismo ocurre con O.bataua con dos en casi todas las comunidades, excepto en San Nandita (V.U.=1.1).

Tabla 6. Valor de uso (V.U.) de las palmeras utilizadas por los Yuracaré.

\begin{tabular}{c|c}
\hline Especie & V.U. \\
\hline Attalea blepharopus & 2.2 \\
\hline Iriartea deltoidea & 2.1 \\
\hline Oenocarpus bataua & 2 \\
\hline Socratea exorrhiza & 1.7 \\
\hline Attalea princeps & 1.7 \\
\hline Astrocaryum gratum & 1.6 \\
\hline Bactris gasipaes & 1.5 \\
\hline Euterpe precatoria & 0.6 \\
\hline Geonoma deversa & 0.3 \\
\hline Hyospathe elegans & 0.1 \\
\hline Mauritia flexuosa & 0.1 \\
\hline Bactris major & 0.1 \\
\hline
\end{tabular}

\section{Categorías de uso y partes utilizadas de las palmeras}

Se identificaron 43 usos diferentes de las palmeras. Algunas especies tienen 2 ó más usos, se agrupan en 22 subcategorías y seis categorías (Tabla 7). Las categorías de uso con más reportes obtenidos son: Alimentación (45\%), construcción con el 38\%, utensilios y herramientas (9\%) (Figura 5). Se obtuvo una descripción cualitativa por categoría y por especie de las diferentes formas de uso de las palmeras utilizadas por las comunidades Yuracaré (Tablas 8 y 9). 
Tabla 7 : Categorías y subcategorías de los usos citados por los entrevistados de las comunidades Yuracaré.

\begin{tabular}{|c|c|c|}
\hline Categorias de uso & Sub-categoría & Uso \\
\hline \multirow{3}{*}{ Alimentación humana } & Alimento & Los frutos de varias especies se comen crudos o cocidos, maduros o inmaduros \\
\hline & Bebidas & Se elaboran bebidas (refresco o leche), fermentadas (chicha) de los frutos o del cogollo \\
\hline & Aceites & Se obtienen grasas comestibles o aceites para freir a partir de frutos y semillas \\
\hline \multirow{3}{*}{ Construcción } & Casas & Los troncos se utilizan para la construcción de las paredes \\
\hline & Techado & Las hojas trenzadas son utilizadas en el techado de casas \\
\hline & Cercos & Los troncos son utilizados en la delimitación de propiedades o como barreras \\
\hline \multirow{3}{*}{ Cultural } & Ropa & Utilizan el cogollo para fabricar sombreros \\
\hline & Cosmético & Utilizan el aceite de las semillas para el cuidado del cabello \\
\hline & Adorno personal & Las semillas son utilizadas para fabricar collares, pulseras y anillos \\
\hline \multirow{8}{*}{ Medicinal y veterinario } & Embarazo, parto y puerperio & La raiz se usa para curar las hemorragias \\
\hline & Enfermedades culturales & El aceite mezclado con tabaco cura el arrebato \\
\hline & Contravenenos & La savia de la raíz actúa como antiofídico y cura la picadura de hormiga (tucandera) \\
\hline & Metabolismo y nutrición & El raspado de raíz ayuda a aumentar de peso \\
\hline & Piel y tejido subcutáneo & La semilla ayuda a curar la caracha, las espinas ayudan en la extracción de nigua (ácaro) \\
\hline & Sangre y sistema circulatorio & El raspado de raiz se utiliza para curar la anemia \\
\hline & $\begin{array}{l}\text { Sintomas sin especificar y } \\
\text { enfermedades generales }\end{array}$ & La savia de raíz y los aceites se utilizan para bajar la fiebre \\
\hline & Sistema digestivo & $\begin{array}{l}\text { La savia de raiz es utilizada para infecciones estomacales, los frutos cocidos se utiliza para } \\
\text { curar desordenes de la vesicula. El raspado de raiz cura la diarrea y actúa como purgante } \\
\text { eliminando parásitos }\end{array}$ \\
\hline Derivados & & El gusano del fruto es utilizado como carnada \\
\hline \multirow{3}{*}{$\begin{array}{l}\text { Utensilios y } \\
\text { herramientas }\end{array}$} & Utensilios domésticos & $\begin{array}{l}\text { Las hojas y el cogollo se utilizan para fabricar canastos, abanicos, cestas, esteras. El tronco } \\
\text { es utilizado para fabricar catres }\end{array}$ \\
\hline & Herramientas de caza y pesca & El tronco y las espinas se utilizan para fabricar arcos y flechas \\
\hline & Herramientas de trabajo & $\begin{array}{l}\text { El tronco de algunas palmeras con espinas se utiliza para fabricar ralladores de yuca, los } \\
\text { troncos más gruesos se utilizan para fabricar tacú o morteros, también para fabricar los } \\
\text { mangos de hachas. Las hojas se utilizan para fabricar escobas }\end{array}$ \\
\hline
\end{tabular}

Figura 5 : Porcentajes de usos reportados en cada categoría.

\begin{tabular}{|c|c|c|c|}
\hline Derivados & $0,1 \%$ & & \\
\hline Utensilios y herramientas & $9 \%$ & & \\
\hline Medicinal & $4 \%$ & & \\
\hline Cultural & $4,2 \%$ & & \\
\hline Construcción & & $37,5 \%$ & \\
\hline Alimentación & & & $45,2 \%$ \\
\hline
\end{tabular}


Tabla 8 : Descripción de los usos de palmeras útiles.

\begin{tabular}{|c|c|c|c|}
\hline \multirow{2}{*}{ Categoria } & \multirow{2}{*}{ Especies } & \multicolumn{2}{|c|}{ Descripción } \\
\hline & & Mujeres & Hombres \\
\hline $\begin{array}{l}\text { Alimentación } \\
\text { humanaa }\end{array}$ & $\begin{array}{l}\text { Aiphanes horrida } \\
\text { Astrocaryum gratum } \\
\text { Attalea blepharopus } \\
\text { Attalea princeps } \\
\text { Bactris gasipaes } \\
\text { Bactris major } \\
\text { Euterpe precatoria } \\
\text { Hyospathe elegans } \\
\text { lriartea deltoidea } \\
\text { Mauritia flexuosa } \\
\text { Oenocarpus bataua } \\
\text { Socratea exorrhiza }\end{array}$ & $\begin{array}{l}\text { Se encargan de cocinar los frutos y el palmito cuando } \\
\text { es necesario, aunque pueden ser consumidos crudos. } \\
\text { Extraen aceite de las semillas y frutos utilizado } \\
\text { en la cocina. De los frutos y el palmito cocidos se } \\
\text { elaboran bebidas refrescantes o fermentadas que } \\
\text { son consumidas en las fiestas (chicha o similar a la } \\
\text { leche). }\end{array}$ & $\begin{array}{l}\text { Se encargan de buscar las mejores palmas } \\
\text { y cosechar los frutos y el palmito. }\end{array}$ \\
\hline Construcción & $\begin{array}{l}\text { Astrocaryum gratum } \\
\text { Attalea blepharopus } \\
\text { Attalea princeps } \\
\text { Euterpe precatoria } \\
\text { Geonoma deversa } \\
\text { Iriartea deltoidea } \\
\text { Oenocarous bataua } \\
\text { Socratea exorrhiza }\end{array}$ & $\begin{array}{l}\text { Utilizan la corteza de las palmas para el tejido de } \\
\text { esteras. }\end{array}$ & $\begin{array}{l}\text { El tronco es utilizado para postes y vigas } \\
\text { en las viviendas, también fabricar catres, } \\
\text { cercos y pisos. Las hojas son cosechadas y } \\
\text { seleccionadas para el techado de viviendas. }\end{array}$ \\
\hline Medicinal & $\begin{array}{l}\text { Astrocaryum gratum } \\
\text { Attalea blepharopus } \\
\text { Bactris gasipaes } \\
\text { Euterpe precatoria } \\
\text { lriartea deltoidea } \\
\text { Oenocarpus bataua } \\
\text { Socratea exorriza }\end{array}$ & $\begin{array}{l}\text { La raiz es preparada para cura la diarrea, ayuda } \\
\text { a parar hemorragias y calmar las picaduras de } \\
\text { hormigas y es un antiofidico. El aceite extraido } \\
\text { ayuda a bajar la fiebre y curar el arrebato. El extracto } \\
\text { de frutos es utilizado para la vesícula. }\end{array}$ & $\begin{array}{l}\text { Preparan la raiz para calmar las picaduras } \\
\text { de hormigas y viboras. }\end{array}$ \\
\hline $\begin{array}{l}\text { Herramientas y } \\
\text { utensilios }\end{array}$ & $\begin{array}{l}\text { Astrocaryum gratum } \\
\text { Attalea blepharopus } \\
\text { Attalea princeps } \\
\text { Bactris gasipaes } \\
\text { Bactris major } \\
\text { Iriartea deltoidea } \\
\text { Socratea exorriza }\end{array}$ & $\begin{array}{l}\text { Las hojas sirven para fabricar escobas, canastos y } \\
\text { abanicos. Las raices fúlcreas son cortadas para ser } \\
\text { utilizadas como rallador. }\end{array}$ & $\begin{array}{l}\text { El tronco es utilizado para fabricar arcos, } \\
\text { flechas, tacú, también sefabrican moledores } \\
\text { de yuca. }\end{array}$ \\
\hline Cultural & $\begin{array}{l}\text { Attalea blepharopus } \\
\text { Attalea princeps } \\
\text { Bactris gasipaes } \\
\text { Chamaedorea angustisecta }\end{array}$ & $\begin{array}{l}\text { El aceite es utilizado para el cuidado del cabello. Las } \\
\text { semillas son utilizadas en la fabricación de collares, } \\
\text { anillos y adornos que utilizan en fiestas locales. }\end{array}$ & \\
\hline Derivados & Attalea princeps & & $\begin{array}{l}\text { Las larvas que se desarrollan en los troncos } \\
\text { son utilizadas como carnadas de pesca. }\end{array}$ \\
\hline
\end{tabular}

Tabla 9 : Descripción de ciertos usos por especie de palmera.

\begin{tabular}{|c|c|c|c|c|c|c|}
\hline Nombre cientifico & Nombre Yuracaré & Fiesta o ritual religioso & Cultivada & Silvestre & Cesteria & Comercio \\
\hline Aiphanes horrida & Kunna & & & $x$ & & - \\
\hline Astrocaryum gratum & Choo & & & $\mathrm{x}$ & Cestas y abanicos & - \\
\hline Attalea blepharopus & Corokke & $\mathrm{x}$ & & $x$ & Cestas y abanicos & - \\
\hline Attalea princeps & Sipe & & & $\mathrm{x}$ & $\begin{array}{l}\text { Canastas, esteras, } \\
\text { abanicos }\end{array}$ & $\begin{array}{l}\text { Ocasionalmente } \\
\text { venden los frutos } \\
\text { entre poblaciones o } \\
\text { llevan a Villa Tunari. }\end{array}$ \\
\hline Bactris gasipaes & Tëmbi & $x$ & $x$ & $x$ & & $\begin{array}{l}\text { Generalmente } \\
\text { comercializan los } \\
\text { frutos cocidos con sal } \\
\text { en Villa Tunari. }\end{array}$ \\
\hline Bactris major & Tërishë & & & $\mathrm{x}$ & & - \\
\hline $\begin{array}{l}\text { Chamaedorea } \\
\text { angustisecta }\end{array}$ & Siyeye & $x$ & & $x$ & & - \\
\hline Euterpe precatoria & Murishi & & $x$ & $x$ & Cestas & $\begin{array}{l}\text { Solo por encargo se } \\
\text { vende el palmito o } \\
\text { los frutos. }\end{array}$ \\
\hline Geonoma deversa & Mushshu & & & $x$ & Abanicos & $\begin{array}{l}\text { Solo por encargo se } \\
\text { comercializan los } \\
\text { paños de las hojas } \\
\text { de jatata. }\end{array}$ \\
\hline Hyospathe elegans & Siribaré & & & $\mathrm{x}$ & & - \\
\hline Iriartea deltoidea & Winna & & & $\mathrm{x}$ & & $\begin{array}{l}\text { Por encargo se } \\
\text { paga por los troncos } \\
\text { seccionados para } \\
\text { construcción. }\end{array}$ \\
\hline Mauritia flexuosa & Shushundala & & $\mathrm{x}$ & $x$ & & - \\
\hline Oenocarpus bataua & Uruppa & & $x$ & $x$ & & $\begin{array}{l}\text { Estacionalmente se } \\
\text { venden los frutos o } \\
\text { se vende el refresco } \\
\text { "leche de majo". }\end{array}$ \\
\hline Socratea exorrhiza & Putërëjtë & & & $x$ & & $\begin{array}{l}\text { Por encargo se paga } \\
\text { por los troncos para } \\
\text { construcción. }\end{array}$ \\
\hline
\end{tabular}

Las partes más utilizadas de las palmeras son los frutos (28\%); consumidos crudos o cocidos (Fotografia 1), también son utilizados para preparar bebidas; los troncos $(20 \%)$ 
son utilizados como pilares de las viviendas, también con ellos se elaboran paredes (Fotografia 2), cercos, catres $^{7}$, herramientas y utensilios. Las semillas (19\%) son utilizadas para extraer aceites para la cocina, cuidado del cabello y uso medicinal, también las hojas (16.5\%) son utilizadas para techados temporal o permanente de viviendas (Fotografia 2) o para la fabricación de escobas; una de las partes menos utilizada es la savia que es exclusivamente de uso medicinal (Figura 6), al igual que la raíz de la cual también se puede obtener herramientas como ralladores (Fotografia 3).

Fotografia 1 : Frutos de Aiphanes horrida (kunna ${ }^{8}$ ).

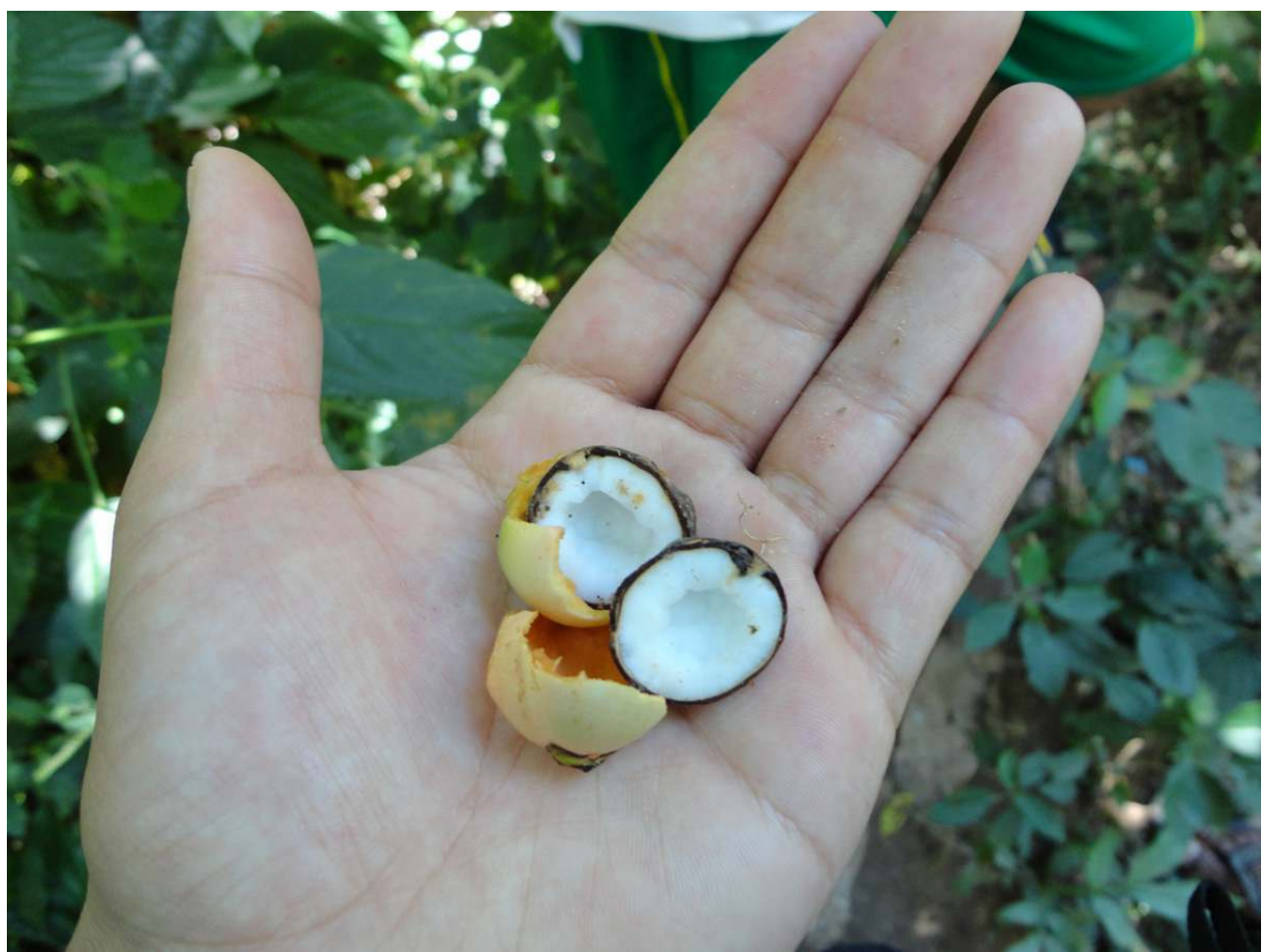


Fotografia 2 : Vivienda con techo de Geonoma deversa (mushshu9 ${ }^{9}$ y paredes de Iriartea deltoidea (winna).

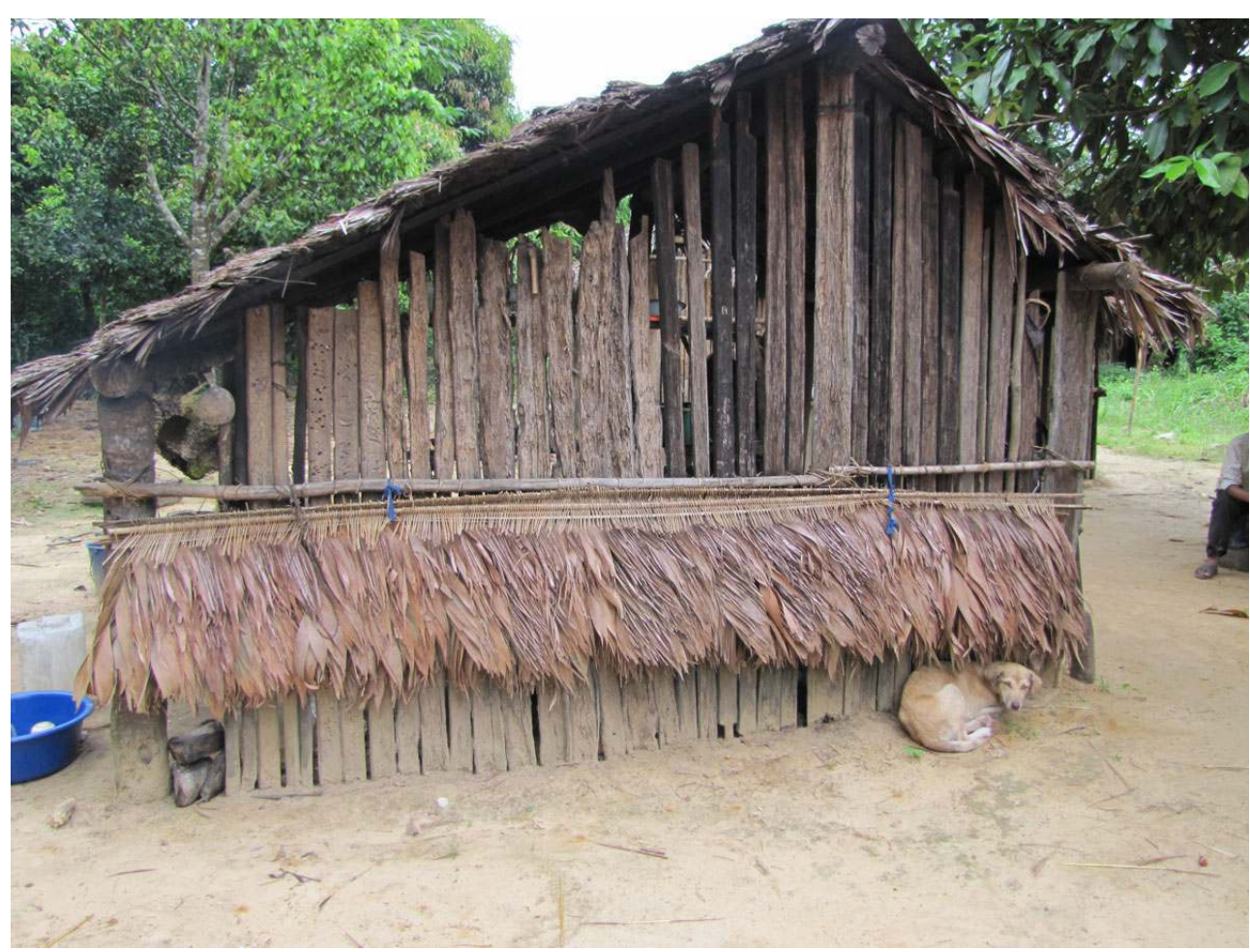

(c) Narel Paniagua

Figura 6 : Partes de las palmeras comunmente conocidas o utilizadas por el pueblo Yuracaré.

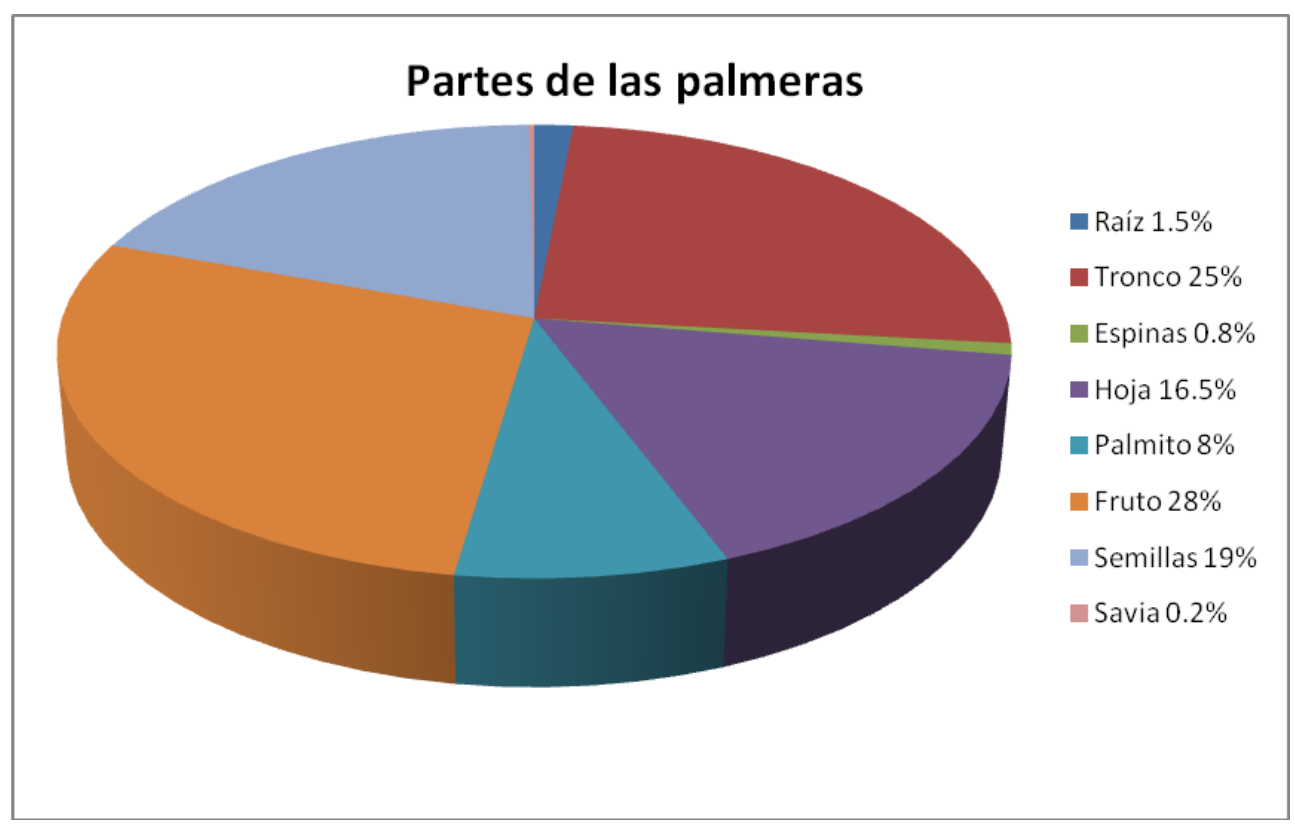




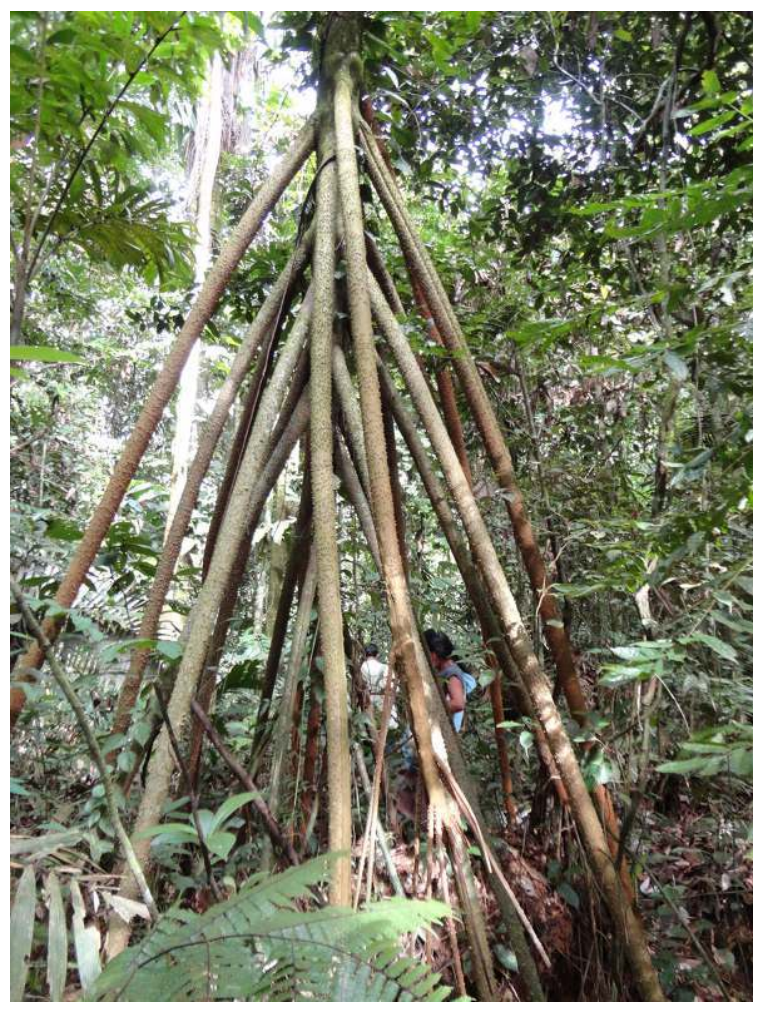

\section{Discusión}

\section{Palmeras útiles por comunidad}

En las cinco comunidades Yuracaré se identificaron en promedio seis palmeras útiles, es decir el 43\% del total de las catorce especies útiles que ocurren en la región. Moraes et al. (1995) registraron para la zona de influencia del territorio Tacana que el 55\% de las palmeras nativas es utilizado para diferentes propósitos. Paniagua (2005a) reportó un 45\% del total de especies presentes en la región del Madidi, mientras que Paniagua et al. (2007) mencionaron que es el $47 \%$ cuando evaluaron los usos de palmas en el oeste amazónico (Bolivia y Perú).

En el presente estudio la comunidad con mayor diversidad de uso es San Antonio, el índice de diversidad de uso (Cinv= 8.85) es significativamente mayor que el resto de las comunidades. Además fue la única comunidad donde todavía se observó el uso cotidiano de utensilios hechos con palmeras (Fotografia 4), el consumo de frutos, la fabricación de anillos, collares y adornos que generalmente son usados por mujeres en las fiestas y ceremonias locales y donde todas las casas tenían pilares, techos o cercos hechos con partes y estructuras de alguna especie, probablemente influenciado por la distancia a centros de comercialización (más de $40 \mathrm{~km}$ ) que es mayor que el resto. Sesecjsama contó con el índice de diversidad más bajo, esta comunidad se encuentra al borde del camino y hay mayor interacción con comunidades colonas y centros de comercialización. Esto indicaría que la marginación o aislamiento de una comunidad, condiciones de los ecosistemas a disposición de cada pueblo y accesos a pueblos más grandes o ciudades son 
factores que afectan el conocimiento y uso de las diferentes comunidades, como argumentaron Paniagua et al. (2007), Miller (2002) y Byg \& Balslev (2001).

Fotografia 4 : Mujer yuracaré mezclando con una paleta hecha con Bactris gasipaes (tëmbi).

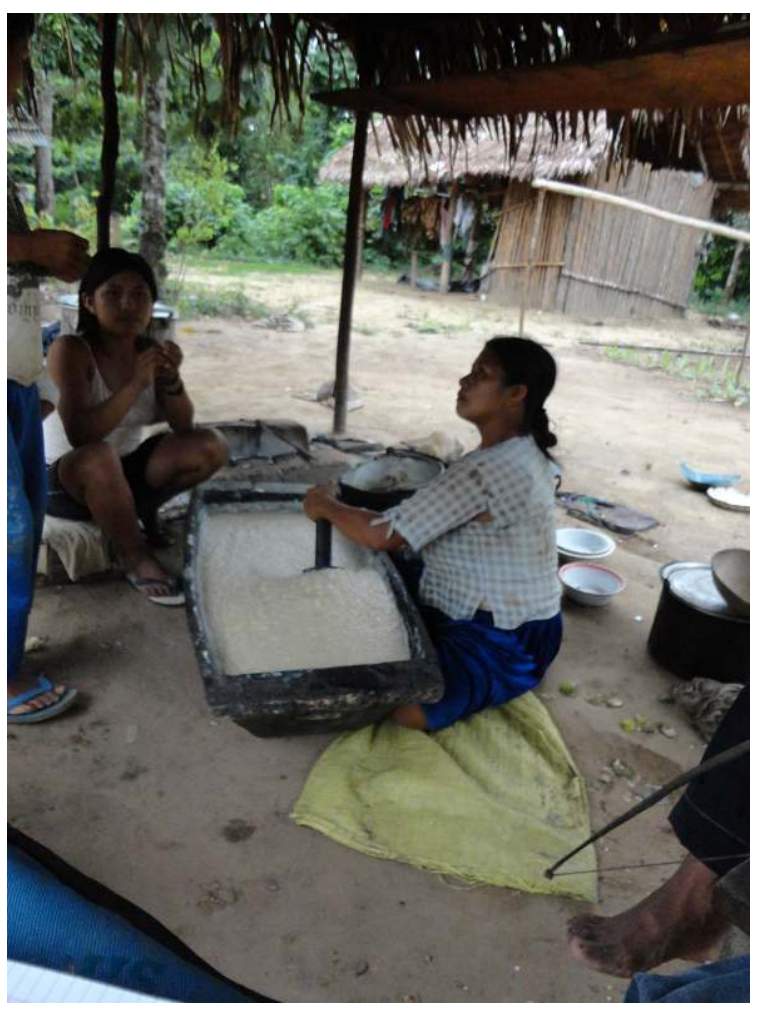

La diversidad de usos en las comunidades estudiadas varía. La palmera con mayor número de usos reportados para San Antonio y San Benito es Bactris gasipaes, para Sesecjsama es Iriartea deltoidea, para San Nandita es Attalea blepharopus y para San Juan, Astrocaryum gratum. Esta variación posiblemente se relaciona con diferencias en la diversidad y concentración en ciertos tipos de usos por comunidad. A la vez podrían relacionarse con la pérdida de oportunidades para aprender, debido a la desaparición de ciertas especies (Anyinam 1995) o debido a la aparición de alternativas para el uso de recursos como materiales de fabricación industrial (Paniagua et al. 2007; Ladio \& Lozada 2004; Byg \& Balslev 2001).

Las curvas de rango-abundancia muestran una equitatividad similar en las cinco comunidades, por lo que la diferencia en índices de diversidad podría atribuirse al número de especies raras. Existe variación en la composición de la comunidad de palmeras, pero tres especies están presentes entre las cinco más abundantes: B. gasipaes, I. deltoidea y A. gratum. Esta variación puede atribuirse al grado de perturbación y el tipo de bosque que las rodea, que influyen en la diversidad de palmeras y vegetación.

\section{Palmeras útiles por categorías de género y edad}

Al realizar estudios etnobotánicos existen ciertas variables culturales y sociales que deben ser tomadas en cuenta, como la edad, el género, el estado social actual, entre otras (Balslev et. al 2008; Paniagua et. al 2007; Kristensen \& Balslev 2003; Martin 2000). No se registraron diferencias significativas al analizar los datos en el conocimiento entre 
hombres y mujeres en el presente estudio. Las diferencias de género en los conocimientos etnobotánicos son a menudo atribuidos a la división de las responsabilidades del hogar, el trabajo y la experiencia en el ámbito intrafamiliar, entre hogares y la comunidad (Paniagua et al. 2007; Aguirre 2006). En la práctica, hombres y mujeres Yuracaré utilizan los recursos y el espacio de una forma diferente, relacionándose con el ambiente que los rodea. Por ejemplo, las mujeres conocen más sobre plantas medicinales y las utilizadas para cocinar, en cambio los hombres generalmente tienen mayor conocimiento de aquellas usadas en la construcción y las comerciales (Paniagua et al. 2007; Byg \& Balslev 2004; Hinojosa et al. 2001). En cuanto a las actividades; los hombres se encargan de la cosecha de frutos y hojas, las mujeres son las encargadas de seleccionar los mejores frutos, prepararlos y cocinarlos si es necesario, también son ellas las que preparan las bebidas a base de frutos o palmito. Las hojas que son empleadas para el techado generalmente son seleccionadas por los hombres, en cambio las mujeres eligen las mejores hojas para la fabricación de escobas, cestos o canastas (Fotografias 5 y 6), abanicos. Los materiales para la elaboración de flechas, arcos (Fotografia 7), mangos de hachas y moledores son seleccionados por los hombres, ellos también se encargan de fabricarlos. Sin embargo, se observó que en muchas ocasiones hombres y mujeres realizan estas actividades en el bosque y comparten los conocimientos sobre cada hábitat. Así por ejemplo en trabajos donde muchas veces se requiere más de una persona, es frecuente que la mujer acompañe a su esposo y lo ayude a realizar diferentes tareas.

Fotografia 5 : Niño Yuracaré sujetando una canasta hecha de Attalea princeps (sipe).

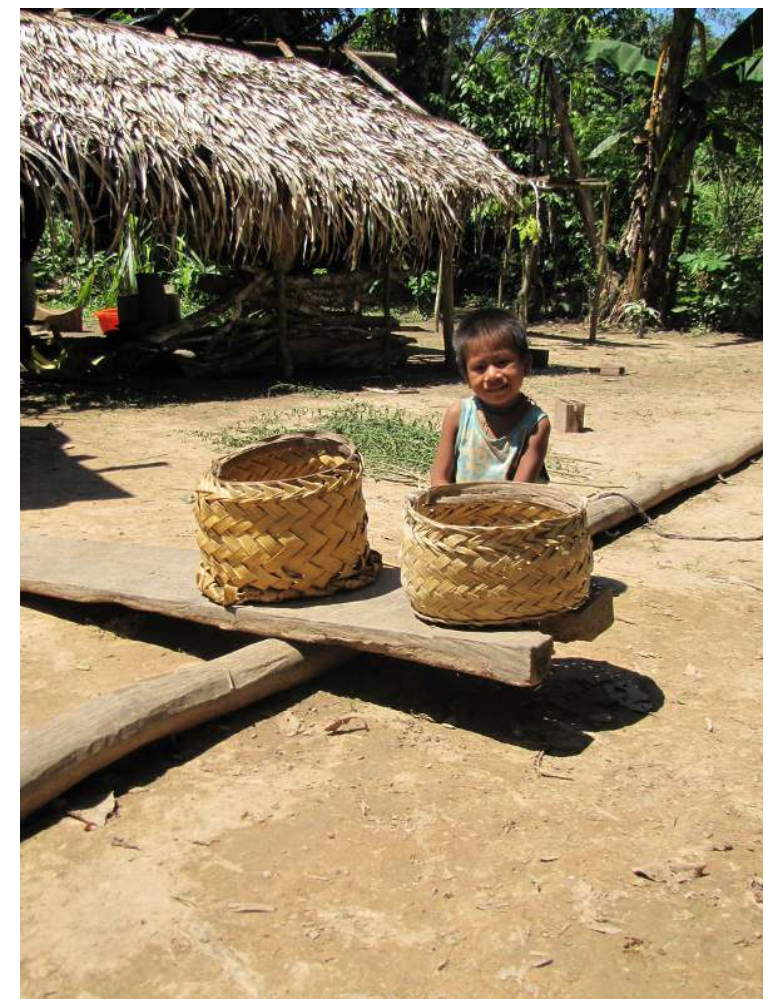

(C) NAREL PANIAgUA 
Fotografia 6 : Cesta hecha con Iriartea deltoidea (winna).

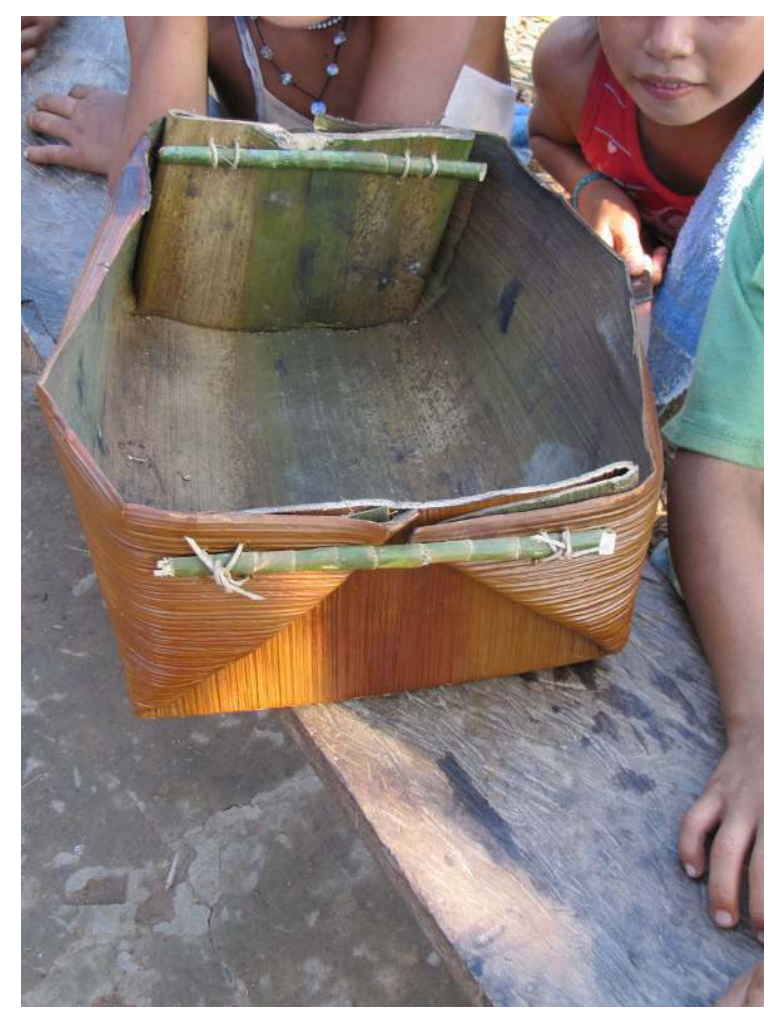

(C) NAREL PANIAgUA

Fotografia 7 : Arco y flecha hechos con Bactris gasipaes (tëmbi).

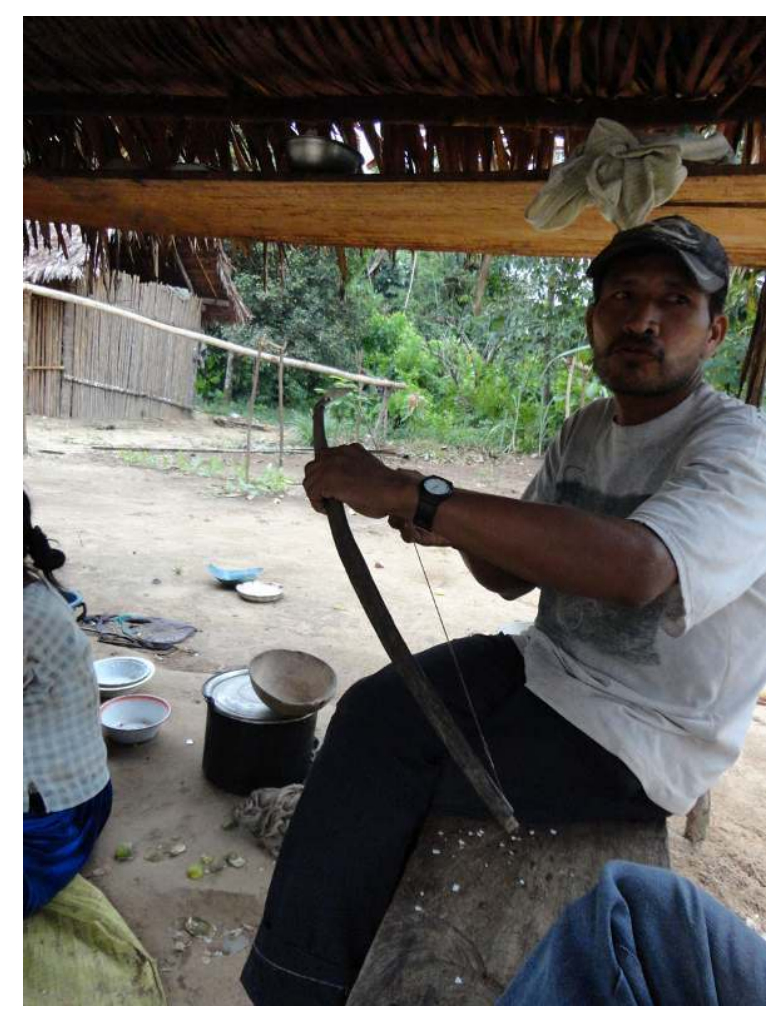


Respecto al uso y conocimiento del uso de palmeras según edad, las personas mayores a los 60 años poseen mayor conocimiento. Aguirre (2006) asevera que las personas con más edad conocen más el uso de sus recursos naturales, al igual que Flores \& Albizú (2005), Sánches (2002), Hinojosa et al. (2001) y Vargas (1997). Aparentemente el conocimiento de las palmeras y sus usos por los informantes más jóvenes proviene de dos fuentes: Los ancianos que transmiten el conocimiento y al ponerse en contacto con su entorno para cubrir las necesidades que surgen en su vida diaria (Paniagua et al. 2007; Kristensen \& Balslev 2003). Sin embargo, el hecho de que los informantes conozcan una especie, no garantiza que sepan cómo se usa o que la usen actualmente.

Hinojosa et al. (2003) mencionaron que actualmente la población Yuracaré es básicamente sedentaria, ahora se relacionan con los colonos, comerciantes y empresarios que han sido un impulso para volver a definir y dimensionar la relación planta-hombre. Esto pudo evidenciarse durante las entrevistas a personas mayores de 60 años que conocían en promedio mayor número de especies útiles, además perciben la pérdida de interés por parte de los más jóvenes en relación a sus tradiciones con el idioma y las costumbres.

\section{Palmeras utilizadas por los Yuracaré}

Iriartea deltoidea, Bactris gasipaes, Astrocaryum gratum y Oenocarpus bataua son las especies más usadas por los entrevistados; dos especies (I. deltoidea y B. gasipaes) son las que presentan mayor cantidad de usos y se encuentran con mayor número de categorías de uso. Además de tener la mayor versatilidad de usos, son las más frecuentemente usadas en la actualidad y desde hace cientos de años, según uno de los indígenas Yuracaré (Daniel Semani com. pers. 2011). Actualmente ellos no manejan ninguna de estas palmeras, utilizan los recursos que provee el bosque, sin embargo ciertas familias Yuracaré tienen palmeras cultivadas en sus patios, especialmente las de uso alimenticio como Bactris gasipaes, Euterpe precatoria (murishi ${ }^{11}$ ) y Oenocarpus bataua. Estas especies son ocasionalmente comercializadas (Fotografia 8).

Fotografia 8 : Comercio de frutos de planta cultivada de Bactris gasipaes (tëmbi), en la comunidad de Sesecjsama.

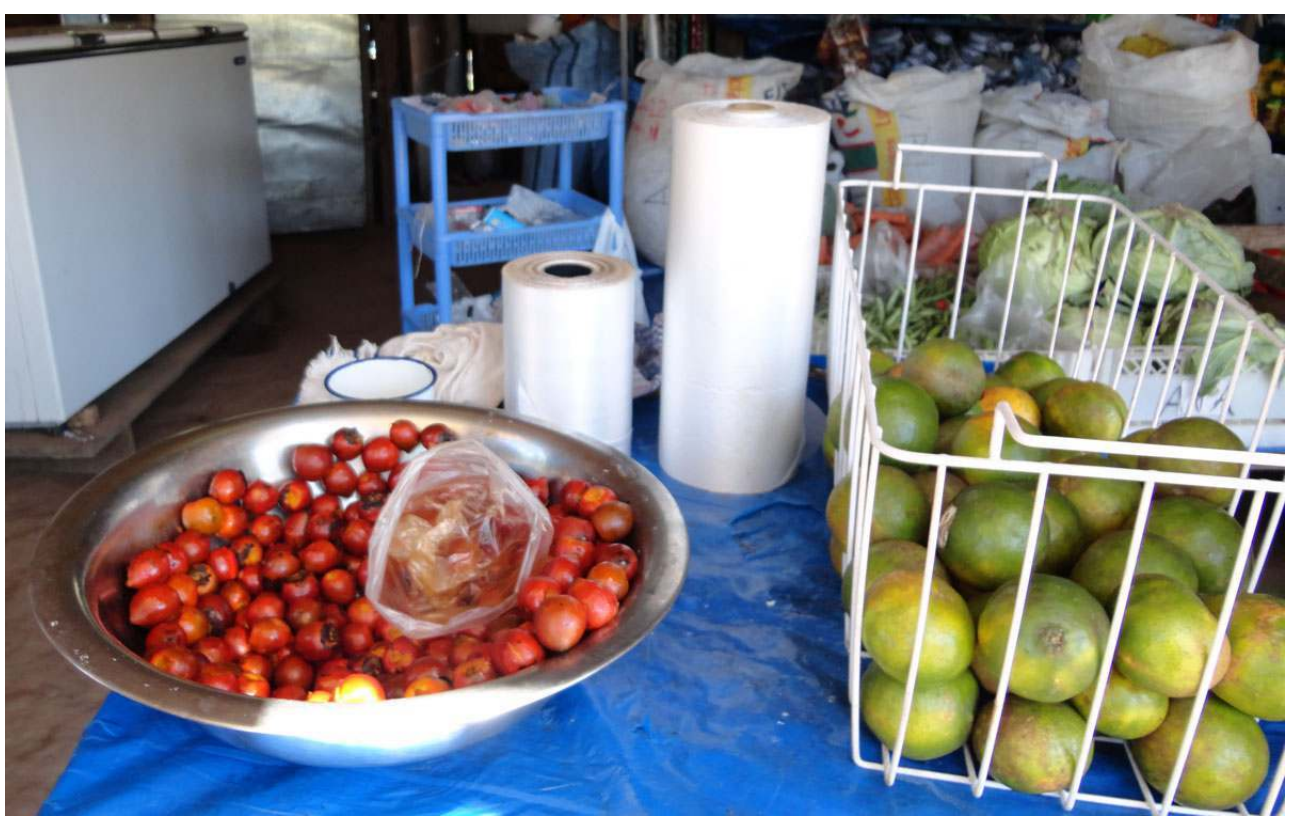



que presentan mayor cantidad de usos de acuerdo a la percepción y consenso de las comunidades Yuracaré. También están las especies cuyos valores de usos son más bajos como M. flexuosa e Hyospathe elegans (siribare ${ }^{13}$ ). Paniagua et al. (2007), Kristensen \& Balslev (2003), Byg \& Balslev (2001) y Hellier et al. (1999) indican que al obtener la percepción de una comunidad sobre la que denominan a una especie como 'importante', da la pauta para lo que se espera obtener. En este caso las tres palmas que son consideradas como las más importantes por la comunidad Yuracaré (A. blepharopus, I. deltoidea y 0 . bataua) tienen mayor cantidad de usos y tienen un mayor valor de uso.

\section{Categorías de uso}

La mayoría de los usos registrado están dentro las categorías de alimentación humana y construcción. Moraes et al. (2014), Ledezma (2011), Balslev et al. (2008), Paniagua et al. (2007) y Kristensen \& Balslev (2003) coinciden con este trabajo, reportando que las categorías de usos más comunes en el oeste de la Amazonía son alimentación, construcción, herramientas y utensilios. Mesa (2011) y De la Torre et al. (2009) también 
reportaron como la categoría de uso más importante la alimenticia, seguida por materiales de construcción y medicinal.

Entre los principales usos del presente estudio y dentro la categoría alimenticia, sobresalen el consumo directo de frutos; crudos o cocidos y la elaboración de bebidas frescas o fermentadas que se preparan para las fiestas locales, que dependiendo de la época, son cosechados. En la categoría construcción; se usan las hojas y los troncos para la construcción de viviendas; ambos son utilizados en cualquier época del año, siempre y cuando sea un individuo maduro y de gran estatura. Los demás usos son igual de importantes como la fabricación de cercos, armazón de la cama, entre otros.

\section{Conclusiones} gratum.
La comunidad que presentó mayor diversidad de usos de palmeras fue San Antonio, en la que todavía se observa el uso cotidiano de utensilios hechos con palmeras, continúan construyendo los techos y pilares de las casas con troncos y hojas de las palmeras. Sesecjsama registró el menor índice de diversidad y menor conocimiento sobre palmeras útiles. Esas diferencias se explican por la relación inversa a la distancia de centros de comercio de colonos, como lo vimos más arriba.

Existe variación en composición de la comunidad de palmeras, grado de perturbación, acceso a servicios, entre otros. Sin embargo, tres especies útiles son comunes y más abundantes en las cinco comunidades: Bactris gasipaes, Iriartea deltoidea y Astrocaryum

El conocimiento en género sobre el uso de palmeras en estas comunidades Yuracaré registra una participación igualitaria entre hombres y mujeres; no obstante muchas mujeres se mostraron tímidas y reacias a compartir información y esto sesga lo proyectado como tendencias en los pueblos originarios. En la comunidad cumplen actividades diferentes: Los varones, se ocupan de conseguir alimentos para la familia, mientras que las mujeres se encargan de la salud y cuidado de los hijos.

Como es de esperarse las personas mayores a los 60 años poseen mayor conocimiento, sin embargo muchas veces se niegan a proporcionar información. La mayoría argumenta que han olvidado sus conocimientos o simplemente no quisieron participar en las entrevistas. El conocimiento que adquieren los más jóvenes proviene de los ancianos y al ponerse en contacto con su entorno para cubrir sus necesidades diarias.

En las cinco comunidades se registró un total de 14 especies de palmeras útiles, las especies que presentan mayor cantidad de usos son: Attalea blepharopus, Iriartea deltoidea, Bactris gasipaes, Astrocaryum gratum y Oenocarpus bataua. Casi el 100\% de los entrevistados conoce el mismo número de usos para todas las especies. Attalea blepharopus es considerada la palmera más importante por los Yuracaré, además es la que registra mayor cantidad de usos reportados; le siguen Iriartea deltoidea y Oenocarpus bataua.

alimentación, construcción, utensilios y herramientas. Muchas de estas especies podrían considerarse potenciales recursos biológicos y entrar en programas de manejo y extracción. Pero varias de las especies importantes se encuentran en baja densidad, lo que dificulta las actividades de cosecha. 


\section{BIBLIOGRAFÍA}

Aguirre G. 2006 - Plantas medicinales utilizadas por los indígenas Moseten - Tsimane' de la comunidad Asunción del Quiquibey, en la RB - TCO Pilón Lajas, Beni, Bolivia. Tesis de licenciatura en biología, La Paz, Universidad Mayor de San Andrés, 49 p.

Amaya K. 2005 - Medicinal plant diversity in the community of Pulquina, Municipality of San Lucas, Nor Cinti Province, Chuquisaca Department (Bolivia). Tesis de maestría en biología, Copenhagen, The Royal Veterinary and Agricultural University, $85 \mathrm{p}$.

Anyinam C. 1995 - Ecology and ethnomedicine: exploring links between current environmental crisis and indigenous medical practice. Social Science \& Medicine 40 (3) : 321-329.

Apaza K. 2008 - Conocimiento y uso de plantas medicinales de los médicos tradicionales del centro de medicina natural "Paya" en el valle de Sorata. Tesis de licenciatura en biología, La Paz, Universidad Mayor de San Andrés, 50 p.

Balslev H., Grandez C., Paniagua N., Moller A. L. \& Hansen S. L., 2008 - Palms (Arecaceae) útiles en los alrededores de Iquitos, Amazonía Peruana. Revista Peruana de Biología 15 (1) : 121-132.

Byg A. \& Balslev H. 2001 - Diversity and use of palms in Zahamena, eastern Madagascar. Biodiversity and Conservation $10: 951-970$.

Byg A. \& Balslev H. 2004 - Factors affecting local knowledge palms in Nangaritza Valley, Southeastern Ecuador. Journal of Ethnobiology 24 (2) : 83-106.

Bocco G., Velazquez A. \& Torres A. 2000 - Ciencia, comunidades indígenas y manejo de recursos naturales. Un caso de investigación participativa en México. Interciencia 25 (2) : 64-70.

Borchsenius F. \& Moraes R.M. 2006 - Palmeras andinas. In Moraes R.M., Øllgaard B., Kvist L. P., Borchsenius F. \& Balslev H. (Ed.) Botánica Económica de los Andes Centrales. La Paz, Herbario Nacional de Bolivia, Universidad Mayor de San Andrés, 412-433.

Campos M. T. \& Ehringhaus C. 2003 - Plant virtues are in the eyes of the beholders: a comparison of known uses among indigenous and folk communities of southwestern Amazonia. Economic Botany 57 : 324-344.

Cardoso G. 2002 - Monografía: Visión general de los pueblos indígenas del trópico de Cochabamba respecto a su desarrollo. Tesis de licenciatura en antropología, La Paz, Universidad Católica Boliviana San Pablo, 55 p.

Cerón C. E. 1995 - Etnobiología de los Cofanes de Dureno. Quito, Museo Ecuatoriano de Ciencias Naturales, 206 p.

CONIYURA (Consejo indígena Yuracaré) 1998 - Plan de manejo de bosque del territorio Indígena Yuracaré. Cochabamba, CERES - FTPP-CONIYURA, 35 p.

De la Torre L., Calvo L. M., Salazar C., Balslev H. \& Borchsenius F. 2009 - Contrasting palm species and use diversity in the Yucatan Peninsula and the Ecuadorian Amazon. Biodiversity Conservation 18 : 2837-2853.

Flores K. \& Albizú M. M. 2005 - Caracterización del uso de plantas en el área de amortiguamiento de la Reserva Biológica Indio Maíz, Nicaragua. Trabajo de diploma, Managua, Universidad Nacional Agraria, $39 \mathrm{p}$. 
Galeano G. \& Bernal R. - 2010. Palmas de Colombia. Guía de campo. Bogotá, Instituto de Ciencias Naturales, Facultad de Ciencias, Universidad Nacional de Colombia, 688 p.

Hellier A., Newton A. \& Ochoa S. 1999 - Use of indigenous knowledge for rapidly assessing trends in biodiversity: a case study from Chiapas, México. Biodiversity and conservation $8: 869-889$.

Hinojosa I., Uzquiano E. \& Flores J. 2001 - Los Yuracaré: su conocimiento, experiencia y la utilización de los recursos vegetales en el Río Chapare. La Paz, Instituto de Ecología, Universidad Mayor de San Andrés, $159 \mathrm{p}$.

Killeen T.J., García E. \& Beck S. G. 1993 - Guía de árboles de Bolivia. Herbario Nacional de Bolivia y Missouri Botanical Garden, La Paz, Quipus srl., 832 p.

Kristensen M. \& Balslev H. 2003 - Perceptions, use and availability of woody plants among the Gourounsi in Burkina Faso. Biodiversity and Conservation 12 : 1715-1739.

Ladio A. \& Lozada M. 2004 - Patterns of use knowledge of wild edible plants in distinct ecological environments: a case of a study of a Mapuche community from northwestern Patagonia.

Biodiversity Conservation 13 : 1153-1173.

Ledezma E. D. 2011 - Etnobotánica de las palmas en las tierras bajas del Pacífico colombiano, con énfasis en la palma cabecinegro (Manicaria saccifera Gaertn.). Tesis de maestría en biología, Bogotá, Universidad Nacional de Colombia, $55 \mathrm{p}$.

Martin G.J. 2000 - Etnobotánica: Manual de métodos. Montevideo, Editorial Nordan-Comunidad, $240 \mathrm{p}$.

McKean S. G. 2003 - Toward sustainable use of palm leaves by a rural community in KwazuluNatal, South Africa. Economic Botany 57 (1) : 65-72.

Medinaceli A \& Aguirre G. 2006 - Medicina tradicional de las comunidades indígenas del río Quiquibey, Bolivia. La Paz, Fundación PRAIA, 135 p.

Mesa L. I. 2011 - Etnobotánica de palmas en la Amazonía colombiana: comunidades indígenas Piacocos del Río Guaviare, como estudio de caso. Bogotá, Tesis de maestría en biología, Universidad Nacional de Colombia, $120 \mathrm{p}$.

Miller L. E. 2002 - The Yuracaré Indians of Eastern Bolivia. Geographical Review 4 (6) : 450-464.

Moraes R. M., Sarmiento J. \& Oviedo E. 1995 - Richness and uses in a diverse palm site in Bolivia. Biodiversity and Conservation $4: 719-727$.

Moraes R. M. 2004a - Flora de palmeras de Bolivia. La Paz, Herbario Nacional de Bolivia, Instituto de Ecología, Carrera de Biología, Universidad Mayor de San Andrés, 231 p.

Moraes R. M. 2004b - Evaluación de las palmeras nativas de Bolivia en relación a sus categorías de utilización. Revista Boliviana de Educación Superior en Ciencias (Reboleds) 3 : 63-70.

Moraes R. M. \& Velarde M. J. 2009 - Frutos del asaí (Euterpe precatoria) para pulpa de refrescos y helados. La Paz, VMABCC - Biodiversity international, Herbario Nacional de Bolivia. Universidad Mayor de San Andrés, 20 p.

Moraes R. M. (Ed.) 2014 - Palmeras útiles de Bolivia - Las especies mayormente aprovechadas para diferentes fines y aplicaciones. La Paz, Herbario Nacional de Bolivia, Universidad Mayor de San Andrés, $170 \mathrm{p}$.

Moraes R. M., Paniagua Zambrana N., Cámara-Leret R., Balslev H. \& Macía J. 2014 - Palmeras útiles de Colombia, Ecuador, Perú y Bolivia. In Balslev H., Macía M. J. \& Navarrete H. (Ed.) Cosecha de palmas en el noroeste de Sudamérica - las bases científicas para su manejo y conservación. Quito, Pontificia Universidad Católica del Ecuador - Proyecto PALMS. 
Paniagua N. 2005a - Diversidad, densidad, distribución y uso de las palmas del Madidi, noreste del departamento de La Paz (Bolivia). Ecología en Bolivia 40 (3) : 265-280.

Paniagua N. 2005b - Factores que determinan el conocimiento y uso de las palmeras en la Amazonia Oeste . Tesis de maestría en biología, Aarhus, Universidad de Aarhus, 23 p.

Paniagua N., Byg A., Svenning J., Moraes M., Grandez C. \& Balslev H. 2007 - Diversity of palm uses in the western Amazon. Biodiversity and Conservation $16: 2771-2787$.

Paniagua N., Macía M. J. \& Cámara-Leret R. 2010 - Protocolo para la toma de datos etnobotánicos de palmeras y variables socioeconómicas en comunidades rurales. Ecología en Bolivia 45 (3) : 44-68.

Pardo de Santayana M. \& Gómez Pellón E. 2003 - Etnobotánica: aprovechamiento tradicional de plantas y patrimonio cultural. Anales Jardín Botánico de Madrid 60 (1) : 171-182.

Phillips O. \& Gentry A. H. 1993 - The useful plants of Tambopata, Perú: I. Statistical hypothesis test with a new quantitative technique. Economic Botany 47 (1) : 15-32.

Posey D.A. 2000 - Biodiversity, genetic resources, and indigenous peoples in Amazonia: (Re) discovering the wealth of traditional resources of natives Amazonians. Oklahoma, Institute for Cultural and Social Anthropology, $20 \mathrm{p}$.

Quintana P. G. \& Vargas L. 1995 - Guía popular de plantas utilizadas por los mosetenes de Covendo, Santa Ana y Muchanes (Alto Beni, Bolivia). La Paz, Fondo Nacional para el Medio Ambiente (FONAMA), 186 p.

Rivera Z. T. 2006 - El conocimiento tradicional en la visión indígena. X Sesión del Comité Intergubernamental sobre la propiedad intelectual y recursos genéticos, conocimiento tradicional y folklore . Ginebra, WIPO, 3 p.

Sánches R. M. 2002 - Estudio etnobotánico de la etnia Yuracaré de Sanandita del territorio indígena Parque Nacional Isiboro Sécure. Tesis de licenciatura en biología, Cochabamba, Universidad Mayor de San Simón, 133 p.

SERNAP (Servicio Nacional de Áreas Protegidas) 2002 - Información técnica del Sistema Nacional de Áreas Protegidas de Bolivia. La Paz. 215 p.

Subcentral TIPNIS (Territorio Indígena Parque Nacional Isiboro Sécure). 2010 - Memoria: Foro Departamental: Territorio Indígena y Parque Nacional Isiboro Sécure. Cochabamba, 74 p.

Vargas L. 1997 - Vida y medicina tradicional de los Mosetenes de Muchanes. Ecología en Bolivia 29 : 19-44.

Vormisto J. 2002 - Palms as rainforest resources: how evenly are they distributed in Peruvian Amazonia? Biodiversity and Conservation $11: 1025-1045$.

\section{NOTAS}

1. La pronunciación y fonética son tambi.

2. Pronunciación y fonética: uinna.

3. Pronunciación y fonética: joroque.

4. Pronunciación y fonética: choó.

5. Pronunciación y fonética: urupa.

6. Pronunciación y fonética: sipe.

7. Catre es el armazón de la cama (l'armature, le cadre du lit) (note de l'éditeur) .

8. Pronunciación y fonética: cunna. 
9. Pronunciación y fonética: musshu.

10. Pronunciación y fonética: putarajta.

11. Pronunciación y fonética: murischi.

12. Pronunciación y fonética: shushúndala.

13. Pronunciación y fonética: siríbare.

\section{RESÚMENES}

El Territorio Indígena Parque Nacional Isiboro Sécure (TIPNIS) es rico en diversidad, además es habitado por pueblos étnicos, entre éstos los Yuracaré. Se realizó un estudio etnobotánico en cinco comunidades Yuracaré. El trabajo consistió en entrevistas semi-estructuradas y colección botánica de palmeras nativas, en que se registró información sobre los usos y conocimientos entre hombres y mujeres de diferentes categorías de edad; además se analizó la diferencia de conocimientos entre las diferentes comunidades Yuracaré. Se registró un total de 14 especies de palmeras útiles, entre las más importantes están Bactris gasipaes, Iriartea deltoidea y Astrocaryum gratum. Se identificaron 43 usos, agrupados en 22 subcategorías y seis categorías: Alimentación humana, construcción, cultural, medicinal, derivados, utensilios y herramientas. La comunidad que presentó mayor diversidad de uso de palmeras fue San Antonio probablemente debido al bajo grado de perturbación y poco acceso a nuevas tecnologías y servicios. El conocimiento entre hombres y mujeres es similar, sin embargo las personas de mayor edad poseen mayor conocimiento sobre usos de palmeras. Los resultados muestran que el pueblo Yuracaré vive y tiene una íntima relación con los bosques que rodean sus comunidades.

The Indigenous Territory National Park Isiboro Secure (TIPNIS in Spanish) is rich in biological diversity, and is inhabited by native groups, among them the Yuracaré. An ethnobotanical study was performed in five Yuracaré communities. The work consisted of semi-structured interviews and botanical collections of native palms, when was information on the uses and knowledge among men and women of different age. We also analyzed the difference of knowledge between different Yuracaré communities. There was a total of 14 species of useful palms, among the most important are Bactris gasipaes, Iriartea deltoidea and Astrocaryum gratum. In total 43 applications were identified, grouped into 22 subcategories and six categories: human food, construction, cultural, medicinal, products, tools and utensils. The community of San Antonio registered the largest diversity of palm uses, probably due to a low degree of disturbance and limited access to new technologies and services. The knowledge among men and women is similar; however the older people still own wisdom about uses of palm trees. The Yuracaré ethnic group lives depending on palm products and shows a close relationship with the surrounding forests where they are settled.

Le Territoire Indigène du Parc National Isiboro Secure (TIPNIS en espagnol) est riche en biodiversité et est habité par des communautés autochtones parmi lesquelles on trouve les Yuracaré. Une étude ethnobotanique a été conduite parmi cinq communautés Yuracaré. Le travail a consisté en des entretiens semi-directifs et des récoltes botaniques de palmiers indigènes pour lesquels nous avons repertorié les usages et les savoirs détenus par les hommes et les femmes de différentes tranches d'âge; de plus nous avons analysé les différences de savoirs entre les différentes communautés Yuracaré. Au total, nous avons enregistré 14 espèces de 
palmiers utiles dont les plus importantes sont : Bactris gasipaes, Iriartea deltoidea et Astrocaryum gratum. Nous avons identifié 43 usages groupés en 22 sous-catégories et six catégories: alimentation des hommes, construction, utilisation culturelle, medecinale, produits dérivés, ustensiles et outils. La communauté qui presente la plus grande diversité d'usages des palmiers, est celle de San Antonio, probablement en raison du faible degré de dégradation et d'un d'accès limité aux nouvelles technologies et aux services. La connaissance est équivalente parmi les hommes et les femmes, cependant les personnes les plus âgées possèdent une meilleure connaissance des usages des palmiers. Les resultats montrent que le peuple Yuracaré vit dans une étroite relation avec les forêts qui environnent ses communautés.

\section{ÍNDICE}

Keywords: Bolivia, ethnobotany, useful palms, Yuracaré ethnic group

Índice geográfico: Bolivie

Población Yuracaré

Mots-clés: ethnobotanique, palmiers utiles

Palabras claves: etnia Yuracaré, etnobotánica, palmeras útiles

\section{AUTORES}

\section{FABIOLA MONTOYA M}

Herbario Nacional de Bolivia, Instituto de Ecología, Universidad Mayor de San Andrés, Casilla 10077 - Correo Central, La Paz, Bolivia

Email: fabiola.montoya.m@gmail.com

\section{MÓNICA MORAES R.}

Herbario Nacional de Bolivia, Instituto de Ecología, Universidad Mayor de San Andrés, Casilla 10077 - Correo Central, La Paz, Bolivia 